\title{
COMPLEX VARIETIES AND HIGHER INTEGRABILITY OF Dir-MINIMIZING $Q$-VALUED FUNCTIONS
}

\author{
EMANUELE NUNZIO SPADARO
}

\begin{abstract}
We give a new contribution in revisiting and extending Almgren's results on $Q$-valued Dir-minimizing functions. We provide new elementary proofs of the following two results: every complex variety is locally the graphs of a Dir-minimizing function; the gradients of Dir-minimizing functions, in principle square-summable, are $p$-integrable for some $p>2$. In the planar case, we find the optimal upper bound for the higher integrability exponent $p$.
\end{abstract}

\section{INTRODUCTION}

Almgren developed the theory of Dir-minimizing multi-valued functions in his big regularity paper [1] as a first step toward the regularity of area-minimizing currents in codimension bigger than 1. Following the pioneering ideas of De Giorgi, the starting point was the approximation of minimal currents via harmonic functions, which are the minimizers of the first non-constant term in the expansion of the area functional: the Dirichlet energy. However, due to the unavoidable phenomenon of branching points as, for example, in the area-minimizing currents induced by complex varieties, he needed to develop the theory of Dir-minimizing $Q$-valued functions, that are multi-valued functions minimizing a suitable Dirichlet energy.

In this paper, following the work in [4], we address two questions on Almegren's $Q$-valued functions: we show that complex varieties are locally graphs of Dirminimizing functions and prove the higher integrability of the gradient of a Dirminimizing $Q$-function.

Theorem 0.1. Let $\mathscr{V} \subseteq \mathbb{C}^{\mu} \times \mathbb{C}^{\nu} \simeq \mathbb{R}^{2 \mu} \times \mathbb{R}^{2 \nu}$ be an irreducible holomorphic variety which is a $Q: 1$-cover of the ball $B_{2} \subseteq \mathbb{C}^{\mu}$ under the orthogonal projection. Then, there exists a Dir-minimizing $Q$-valued function $f \in W^{1,2}\left(B_{1}, \mathcal{A}_{Q}\left(\mathbb{R}^{2 \nu}\right)\right)$ such that $\operatorname{graph}(f)=\mathscr{V} \cap\left(B_{1} \times \mathbb{C}^{\nu}\right)$.

Theorem 0.2. There exists $p=p(n, m, Q)>2$ such that, for every $\Omega \subseteq \mathbb{R}^{m}$ open and $u \in W^{1,2}\left(\Omega, \mathcal{A}_{Q}\left(\mathbb{R}^{n}\right)\right)$ Dir-minimizing, $|D u| \in L_{l o c}^{p}(\Omega)$.

Theorem 0.1 provides many examples of Dir-minimizing functions and, in particular, shows that the Hölder continuity and the estimate of the singular set of a Dir-minimizer proved in [1] and [4] are optimal results. Theorem 0.1 has been proved by Almgren in his big regularity paper [1, Theorem 2.20] using a deep and complicated approximation theorem of minimal currents via graphs of Lipschitz $Q$-functions (see also [3]). Here we give a much more elementary proof which relies on a comparison argument and the well-known observation due to Federer that irreducible complex varieties are locally area-minimizing currents. For the planar case, moreover, we also provide an alternative proof which exploits the equality between the area and the energy for conformal maps. We think that this approach 
can be extended to the study of regularity issues for more complicated calibrated geometries.

Theorem 0.2 has been first proved by De Lellis and the author in [3] in connection with a new higher integrability estimate for minimal currents and it plays a crucial role in the proof of Almgren's approximation theorem given there. Here, we propose a different "intrinsic" proof, where "intrinsic" means based only on the metric theory of $Q$-valued functions as developed in [4]. For what concerns the case $m=2$, we found an explicit upper bound for the integrability exponent: using the examples provided by complex varieties in the first part of the paper, we can show that this upper bound is in fact optimal.

The paper is organized as follows. In the first section we collect the basic results and definition on $Q$-valued functions. Then, in Section 2 we prove some results on the push-forward of rectifiable currents under $Q$-valued functions, among which, in particular, a characterization the boundary of the graph of a Lipschitz $Q$-function. In Section 3 we identify complex varieties as graphs of Sobolev $Q$-valued functions and prove Theorem 0.1. Finally, Section 4 contains the proof of Theorem 0.2 which passes through a Caccioppoli and a reverse Hölder inequality for Dir-minimizing functions.

0.1. Acknowledgements. The author is grateful to Camillo De Lellis for many stimulating discussions. This research has been supported by the Forschungskredit of the University of Zurich.

\section{1. $Q$-VALUED FUnCTIONS}

In what follows, we adopt the notation and the approach introduced in [4], which differs from Almgren's original one. For the definitions of the metric space of $Q$ points $\left(\mathcal{A}_{Q}, \mathcal{G}\right)$, Sobolev $Q$-valued function and Dirichlet energy, we refer to [4]. We say that a function $f: \Omega \subset \mathbb{R}^{m} \rightarrow \mathcal{A}_{Q}\left(\mathbb{R}^{n}\right)$ has a smooth local selection in $\Omega^{\prime} \subseteq \Omega$ if, for every $x \in \Omega^{\prime}$, there exist $r>0$ and $f_{i}: B_{r}(x) \rightarrow \mathbb{R}^{n}$ smooth functions such that $\left.f\right|_{B_{r}(x)}=\sum_{i=1}^{Q} \llbracket f_{i} \rrbracket$. Note that, in this case, $|D f|^{2}=\sum_{i}\left|D f_{i}\right|^{2}$ is well defined on the whole $\Omega^{\prime}$. We observe the following simple consequence of the definition, which for reader's convenience we state as a lemma.

Lemma 1.1. Let $f: \Omega \subset \mathbb{R}^{m} \rightarrow \mathcal{A}_{Q}$ have a smooth local selection in $\Omega^{\prime} \subseteq \Omega$. If $\operatorname{dim}_{\mathcal{H}}\left(\Omega \backslash \Omega^{\prime}\right) \leq m-2$ and $\int_{\Omega^{\prime}}|D f|^{2}<+\infty$, then $f$ belongs to $W^{1,2}\left(\Omega, \mathcal{A}_{Q}\right)$.

Proof. The proof follows from the characterization of classical Sobolev functions via the slice property. Indeed, for every $T \in \mathcal{A}_{Q}$, the function $x \mapsto \mathcal{G}(f(x), T)$ is smooth and satisfies $|D(\mathcal{G}(f(\cdot), T))| \leq|D f|$ in $\Omega^{\prime}$ (cp. to [4, Proposition 2.17]). Therefore, since the projection of $\Omega \backslash \Omega^{\prime}$ on each coordinate hyperplane is a set of $\mathcal{H}^{m-1}$ measure zero, for $\mathcal{H}^{m-1}$-a.e. line parallel to the axes, the restriction of $\mathcal{G}(f(\cdot), T)$ belongs to $W^{1,2}$. Recalling [5, Section 4.9.2], it follows that $\mathcal{G}(f(\cdot), T) \in W^{1,2}(\Omega)$ with $|D(\mathcal{G}(f(\cdot), T))| \leq|D f|$ a.e. in $\Omega$. By the definition of Sobolev $Q$-functions [4, Definition 0.5], we, hence, conclude.

We will need also a technical result about the lower semicontinuity of the $L^{p}$ norm of the gradient under weak convergence. Although this is a special case of the result in [2], we include here an elementary proof for the sake of completeness. 
Lemma 1.2 (Semicontinuity). Let $f_{k}, f \in W^{1, p}\left(\Omega, \mathcal{A}_{Q}\right), p<\infty$, be such that $\lim _{k} \int_{\Omega} \mathcal{G}\left(f_{k}, f\right)^{p}=0$ and $\sup _{k} \int_{\Omega}\left|D f_{k}\right|^{p}<\infty$. Then,

$$
\int_{\Omega}|D f|^{p} \leq \liminf _{k \rightarrow+\infty} \int_{\Omega}\left|D f_{k}\right|^{p} .
$$

Proof. The proof of this result is very similar to the proof of the semicontinuity for the Dirichlet energy given in [4, Section 2.3.2]. Let $\left\{T_{l}\right\}_{l \in \mathbb{N}}$ be any dense subset of $\mathcal{A}_{Q}$ and recall that by [4, Proposition 4.2] $|D f|$ is the monotone limit of $h_{N}$ with

$$
h_{N}^{2}=\max _{l_{j} \leq N} \sum_{j}\left(\partial_{j} \mathcal{G}\left(f, T_{l_{j}}\right)\right)^{2} .
$$

By the Monotone Convergence Theorem, $\int|D f|^{p}=\sup _{N} \int h_{N}^{p}$. Therefore, denoting by $\mathcal{P}_{N^{m}}$ the collections $P=\left\{E_{\bar{l}}\right\}_{\bar{l}=\left\{l_{1}, \ldots, l_{m}\right\} \in N^{m}}$ of $N^{m}$ disjoint open subsets of $\Omega$, as in [4] we conclude that

$$
\int_{\Omega}|D f|^{p}=\sup _{N} \int_{\Omega} h_{N}^{p}=\sup _{N} \sup _{P \in \mathcal{P}_{N} m} \sum_{E_{\bar{l}} \in P} \int_{E_{\bar{l}}}\left(\sum_{j}\left(\partial_{j} \mathcal{G}\left(f, T_{l_{j}}\right)\right)^{2}\right)^{\frac{p}{2}} .
$$

It follows easily from the hypotheses that, for every $\bar{l}=\left\{l_{1}, \ldots, l_{m}\right\}$ and every open set $E_{\bar{l}}$, the vector-valued maps $\left(\partial_{1} \mathcal{G}\left(f_{k}, T_{l_{1}}\right), \ldots, \partial_{m} \mathcal{G}\left(f_{k}, T_{l_{m}}\right)\right)$ converge weakly in $L^{p}\left(E_{\bar{l}}\right)$ to $\left(\partial_{1} \mathcal{G}\left(f, T_{l_{1}}\right), \ldots, \partial_{m} \mathcal{G}\left(f, T_{l_{m}}\right)\right)$. Hence, by the semicontinuity of the norm,

$$
\int_{E_{\bar{l}}}\left(\sum_{j}\left(\partial_{j} \mathcal{G}\left(f, T_{l_{j}}\right)\right)^{2}\right)^{\frac{p}{2}} \leq \liminf _{k \rightarrow+\infty} \int_{E_{\bar{l}}}\left(\sum_{j}\left(\partial_{j} \mathcal{G}\left(f_{k}, T_{l_{j}}\right)\right)^{2}\right)^{\frac{p}{2}} .
$$

Summing in $E_{l} \in P$, in view of (1.2), we achieve (1.1).

The main regularity results for Dir-minimizing $Q$-valued functions are collected in the following theorem (see [4, Theorems 0.9 and 0.11]). In order to state them, we recall the definition of regular and singular points.

Definition 1.3. A $Q$-valued function $f$ is regular at a point $x \in \Omega$ if there exist a neighborhood $U$ of $x$ and $Q$ analytic functions $f_{i}: U \rightarrow \mathbb{R}^{n}$ such that $\left.f\right|_{U}=\sum_{i} \llbracket f_{i} \rrbracket$ and either $f_{i}(y) \neq f_{j}(y)$ for every $y \in U$ or $f_{i} \equiv f_{j}$. The singular set $\Sigma_{f}$ of $f$ is the complement of the set of regular points.

Theorem 1.4. For every Dir-minimizing $f \in W^{1,2}\left(\Omega, \mathcal{A}_{Q}\right)$ the following holds:

(i) there exists $\alpha=\alpha(m, Q)>0(\alpha(2, Q)=1 / Q)$ such that $f \in C^{0, \alpha}\left(\Omega^{\prime}\right)$ for every $\Omega^{\prime} \subset \subset \Omega$ and

$$
\operatorname{Dir}\left(f, B_{r}(x)\right) \leq\left(\frac{r}{\rho}\right)^{2 \alpha} \operatorname{Dir}\left(f, B_{\rho}(x)\right), \quad \forall r \leq \rho \text { with } B_{\rho}(x) \subseteq \Omega ;
$$

(ii) the Hausdorff dimension of $\Sigma_{f}$ is at most $m-2$ and, if $m=2, \Sigma_{f}$ consists of isolated points.

\section{Push-Forward of Currents under $Q$-Functions}

The aim of this section is to define the integer rectifiable current associated to the graph of a $Q$-valued function.

Given a $Q$-valued function $f: \mathbb{R}^{m} \rightarrow \mathcal{A}_{Q}\left(\mathbb{R}^{n}\right)$, we set $\bar{f}=\sum_{i} \llbracket\left(x, f_{i}(x)\right) \rrbracket$, $\bar{f}: \mathbb{R}^{m} \rightarrow \mathcal{A}_{Q}\left(\mathbb{R}^{m+n}\right)$. If $R \in \mathscr{D}_{k}\left(\mathbb{R}^{m}\right)$ is a rectifiable current associated to a 
$k$-rectifiable set $M$ with multiplicity $\theta, R=\tau(M, \theta, \xi)$, where $\xi$ is a borel simple $k$-vector field orienting $M$ (we use the notation in [8]), and if $f$ is a proper Lipschitz $Q$-valued function, we can define the push-forward of $T$ under $f$ as follows.

Definition 2.1. Given $R=\tau(M, \theta, \xi) \in \mathscr{D}_{k}\left(\mathbb{R}^{m}\right)$ and $f \in \operatorname{Lip}\left(\mathbb{R}^{m}, \mathcal{A}_{Q}\left(\mathbb{R}^{n}\right)\right)$ as above, we denote by $T_{f, R}$ the current in $\mathbb{R}^{m+n}$ defined by

$$
\left\langle T_{f, R}, \omega\right\rangle=\int_{M} \theta \sum_{i}\left\langle\omega \circ \bar{f}_{i}, D^{M} \bar{f}_{i \#} \xi\right\rangle d \mathcal{H}^{k} \quad \forall \omega \in \mathscr{D}^{k}\left(\mathbb{R}^{m+n}\right),
$$

where $\sum_{i} \llbracket D^{M} \bar{f}_{i}(x) \rrbracket$ is the differential of $\bar{f}$ restricted to $M$.

Remark 2.2. Note that, by Rademacher's theorem [4, Theorem 1.13] the derivative of a Lipschitz $Q$-function is defined a.e. on smooth manifolds and, hence, also on rectifiable sets.

As a simple consequence of the Lipschitz decomposition in [4, Proposition 1.6], there exist $\left\{E_{j}\right\}_{j \in \mathbb{N}}$ closed subsets of $\Omega$, positive integers $k_{j, l}, L_{j} \in \mathbb{N}$ and Lipschitz functions $f_{j, l}: E_{j} \rightarrow \mathbb{R}^{n}$, for $l=1, \ldots, L_{j}$, such that

$$
\mathcal{H}^{k}\left(M \backslash \cup_{j} E_{j}\right)=0 \quad \text { and }\left.\quad f\right|_{E_{j}}=\sum_{l=1}^{L_{j}} k_{j, l} \llbracket f_{j, l} \rrbracket .
$$

From the definition, $T_{f, R}=\sum_{j, l} k_{j, l} \bar{f}_{j, l \#}\left(R\left\llcorner E_{j}\right)\right.$ is a sum of rectifiable currents defined by the push-forward under single-valued Lipschitz functions. Therefore, it follows that $T_{f, R}$ is rectifiable and coincides with $\tau\left(\bar{f}(M), \theta_{f}, \vec{T}_{f}\right)$, where

$$
\theta_{f}\left(x, f_{j, l}(x)\right)=k_{j, l} \theta(x) \quad \text { and } \quad \vec{T}_{f}\left(x, f_{j, l}(x)\right)=\frac{D^{M} \bar{f}_{j, l \#} \xi(x)}{\left|D^{M} \bar{f}_{j, l \#} \xi(x)\right|} \quad \forall x \in E_{j} .
$$

By the standard area formula, using the above decomposition of $T_{f, R}$, we get an explicit expression for the mass of $T_{f, R}$ :

$$
\mathbf{M}\left(T_{f, R}\right)=\int_{M}|\theta| \sum_{i} \sqrt{\operatorname{det}\left(D^{M} \bar{f}_{i} \cdot\left(D^{M} \bar{f}_{i}\right)^{T}\right)} d \mathcal{H}^{k} .
$$

2.1. Boundaries of Lipschitz $Q$-valued graphs. With a slight abuse of notation, when $R=\llbracket \Omega \rrbracket \in \mathscr{D}_{m}\left(\mathbb{R}^{m}\right)$ is given by the integration over a Lipschitz domain $\Omega \subset \mathbb{R}^{m}$ of the standard $m$-vector $\vec{e}=e_{1} \wedge \cdots \wedge e_{m}$, we write simply $T_{f, \Omega}$ for $T_{f, R}$. The same we do for $T_{f, \partial \Omega}$, understanding that $\partial \Omega$ is oriented as the boundary of $\llbracket \Omega \rrbracket$. The main result for what concerns the push-forward under $Q$-valued functions is given in the following theorem.

Theorem 2.3. For every $\Omega$ Lipschitz domain and $f \in \operatorname{Lip}\left(\Omega, \mathcal{A}_{Q}\right), \partial T_{f, \Omega}=T_{f, \partial \Omega}$.

In order to prove this theorem, we need the following slight variant of [4, Homotopy Lemma 1.8].

Lemma 2.4. There exists a constant $c_{Q}$ with the following property. For every $C \subset \mathbb{R}^{m}$ closed cube centered at $x_{0}$ and $u \in \operatorname{Lip}\left(C, \mathcal{A}_{Q}\right)$ Lipschitz, there exists $h \in \operatorname{Lip}\left(C, \mathcal{A}_{Q}\right)$ with the following properties:

(i) $\left.h\right|_{\partial C}=\left.u\right|_{\partial C}, \operatorname{Lip}(h) \leq c_{Q} \operatorname{Lip}(u)$ and $\|\mathcal{G}(u, h)\|_{L^{\infty}} \leq c_{Q} \operatorname{Lip}(u) \operatorname{diam}(C)$;

(ii) $u=\sum_{j=1}^{J} \llbracket u_{j} \rrbracket$ and $h=\sum_{j=1}^{J} \llbracket h_{j} \rrbracket$, for some $J \geq 1$, and $T_{h_{j}, C}$ is a cone over $T_{u_{j}, \partial C}$,

$$
T_{h_{j}, C}=\llbracket\left(x_{0}, a_{j}\right) \rrbracket \nVdash T_{u_{j}, \partial C}, \text { for some } a_{j} \in \mathbb{R}^{n} .
$$


Proof. The proof is essentially contained in [4, Lemma 1.8]. Indeed, $(i)$ follows straightforwardly from the conclusions there. For what concerns (ii), following the inductive argument in [4, Lemma 1.8], due to the obvious invariances it is enough to prove that, for the cone-like extension of $u, h(x)=\sum_{i} \llbracket\|x\| u_{i}(x /\|x\|) \rrbracket$, where $\|x\|=\sup _{i}\left|x_{i}\right|$ is the uniform norm, $T_{h, C_{1}}=\llbracket 0 \rrbracket \times T_{u, \partial C_{1}}$, with $C_{1}=[-1,1]^{m}$. This follows easily from the decomposition $T_{u, \partial C_{1}}=\sum_{j, l} k_{j, l} \bar{u}_{j, l \#}\left(R\left\llcorner E_{j}\right)\right.$ described in the previous subsection. Indeed, setting

$$
F_{j}=\left\{t x: x \in E_{j}, 0 \leq t \leq 1\right\},
$$

clearly $h$ decomposes in $F_{j}$ as $u$ in $E_{j}$ and $\bar{h}_{j, l \#}\left(R\left\llcorner F_{j}\right)=\llbracket 0 \rrbracket \nVdash \bar{u}_{j, l \#}\left(R\left\llcorner E_{j}\right)\right.\right.$.

Proof of Theorem 2.3. Observe that we can reduce to the case the domain $\Omega$ is the unit cube $[0,1]^{m}$. Indeed, by a partition of unity argument, we can assume that there exists $\phi: \Omega \rightarrow[0,1]^{m}$ biLipschitz homeomorphism. Set $g:[0,1]^{m} \rightarrow \mathcal{A}_{Q}$ such that $g \circ \phi=f$ and $\tilde{\phi}(x, y)=(\phi(x), y), \tilde{\phi}: \Omega \times \mathbb{R}^{n} \rightarrow[0,1]^{m} \times \mathbb{R}^{n}$. Hence, following [8, Remark $27.2(3)]$ and using the characterization $T_{f, \Omega}=\tau\left(f(\Omega), \theta_{f}, \vec{T}_{f}\right)$, it is simple to verify that $\tilde{\phi}_{\#} T_{f, \Omega}=T_{g,[0,1]^{m}}$ and analogously $\tilde{\phi}_{\#} T_{f, \partial \Omega}=T_{g, \partial[0,1]^{m}}$. So, since the boundary and the push-forward commute, from now on, without loss of generality, we can assume $\Omega=[0,1]^{m}$.

The proof is by induction on the dimension of the domain $m$. For $m=1$, by the Lipschitz selection principle (cp. to [4, Proposition 1.2]) there exist single-valued Lipschitz functions $f_{i}$ such that $f=\sum_{i} \llbracket f_{i} \rrbracket$. Hence, it is immediate to verify that

$$
\partial T_{f, \Omega}=\sum_{i} \partial T_{f_{i}, \Omega}=\sum_{i}\left(\delta_{f_{i}(1)}-\delta_{f_{i}(0)}\right)=T_{f \mid \partial \Omega} .
$$

For the inductive argument, consider the dyadic decompositions of scale $2^{-l}$ of $\Omega$,

$$
\Omega=\bigcup_{k \in\left\{0, \ldots, 2^{l}-1\right\}^{m}} Q_{k, l}, \quad \text { with } \quad Q_{k, l}=2^{-l}\left(k+[0,1]^{m}\right) .
$$

In each $Q_{k, l}$, set $h_{k, l}$ the cone-like extension given by Lemma 2.4 and

$$
T_{l}=\sum_{k} T_{h_{k, l}, Q_{k, l}}=T_{h_{l}}
$$

with $h_{l}$ the $Q$-function which coincides with $h_{k, l}$ in $Q_{k, l}$. Note that the $h_{l}$ 's are equi-Lipschitz and converge uniformly to $f$ by Lemma $2.4(i)$.

By inductive hypothesis, since each face $F$ of $\partial Q_{k, l}$ is a $(m-1)$-dimensional cube, $\partial T_{f, F}=T_{f, \partial F}$. Taking into account the orientation of $\partial F$ for each face, it follows immediately that

$$
\partial T_{f, \partial Q_{k, l}}=0 .
$$

Moreover, by Lemma 2.4, each $T_{h_{k, l}, Q_{k, l}}$ is a sum of cones. Therefore, using (2.4) and $\partial(\llbracket 0 \rrbracket \nVdash T)=T-\llbracket 0 \rrbracket \nVdash \partial T$ (see $\left[8\right.$, Section 26]), $\partial\left(T_{l}\left\llcorner Q_{k, l}\right)=\partial T_{h_{k, l}, Q_{k, l}}=\right.$ $T_{f, \partial Q_{k, l}}$. Considering the different orientations of the boundary faces of adjacent cubes, it follows that all the contributions cancel except those at the boundary of $\Omega$, thus giving $\partial T_{l}=T_{f, \partial \Omega}$.

The integer $m$-rectifiable currents $T_{l}$, hence, have all fix boundary and equibounded mass (from (2.3), being the $h_{l}$ 's equi-Lipschitz). By the compactness theorem for integral currents (see [8, Theorem 27.3]), there exists an integral current $S$ which is the weak limit for a subsequence of the $T_{l}$ (not relabeled). Clearly, $\partial S=\lim _{l \rightarrow \infty} \partial T_{l}=T_{f, \partial \Omega}$. We claim that $T_{f, \Omega}=S$, thus concluding the proof. 
To show the claim, notice that, since $h_{l} \rightarrow f$ in $L^{\infty}$, then $\operatorname{supp}(S) \subseteq \operatorname{graph}(f)$. So, we need only to show that the multiplicity of the currents $S$ and $T_{f, \Omega}$ coincide almost everywhere. Consider a point $x \in E_{j}$, for some $E_{j}$ in (2.2). From the Lipschitz continuity of $f$ and $h_{l}$, in a neighborhood $U$ of $x, h_{l}$ and $S$ can be decomposed in the same way as $f$,

$$
\left.h_{l}\right|_{U}=\sum_{p=1}^{L_{j}} \llbracket h_{l, p} \rrbracket \quad \text { and } \quad S\left\llcorner\left(U \times \mathbb{R}^{n}\right)=\sum_{p=1}^{L_{j}} S_{p},\right.
$$

where the $h_{l, p}$ 's are $k_{j, p^{-}}$valued and the $S_{p}$ are integer rectifiable $m$-currents with disjoint supports. By definition, the density of $T_{f, \Omega}$ in $\left(x, f_{j, p}(x)\right)$ is $k_{j, p}$. On the other hand, since

$$
\pi_{\sharp} S_{p}=\lim _{l} \pi_{\sharp} T_{h_{l, p}, U}=k_{j, l} \llbracket U \rrbracket \quad \text { and } \operatorname{supp}\left(S_{p}\right) \cap\left(\{x\} \times \mathbb{R}^{n}\right)=\left(x, f_{j, p}(x)\right),
$$

it follows that the density of $S_{p}$ (and hence of $S$ ) in $\left(x, f_{j, p}(x)\right)$ equals $k_{j, p}$. Since $\left|\Omega \backslash \cup_{j} E_{j}\right|=0$, this implies $S=T_{f, \Omega}$.

2.2. First order expansion of the mass. Up to now we have defined pushforward under Lipschitz maps. Nevertheless, thanks to the approximate differentiability property of Sobolev $Q$-functions (see [4, Corollary 2.7]), for full dimensional current $R=\llbracket \Omega \rrbracket$, the definition of $T_{f, \Omega}$ in (2.1) makes sense for Sobolev functions as soon as the action is finite for every differential form $\omega \in \mathscr{D}^{m}\left(\mathbb{R}^{m+n}\right)$. It is easy to verify that this condition is satisfied if

$$
\mathbf{M}\left(T_{f, \Omega}\right)=\int_{\Omega} \sum_{i} \sqrt{\operatorname{det}\left(D^{M} \bar{f}_{i} \cdot\left(D^{M} \bar{f}_{i}\right)^{T}\right)}<+\infty .
$$

For such functions, we have the following Taylor expansion of the mass of $T_{f, \Omega}$.

Lemma 2.5. Let $f \in W^{1,2}\left(\Omega, \mathcal{A}_{Q}\right)$ such that $\mathbf{M}\left(T_{f, \Omega}\right)<+\infty$. Then,

$$
\mathbf{M}\left(T_{\lambda f, \Omega}\right)=Q|\Omega|+\frac{\lambda^{2}}{2} \operatorname{Dir}(f, \Omega)+o\left(\lambda^{2}\right) \quad \text { as } \quad \lambda \rightarrow 0 .
$$

Proof. For every $\lambda>0$, set $A_{\lambda}=\left\{|D f| \leq \lambda^{-\frac{1}{2}}\right\}$ and $B_{\lambda}=\left\{|D f|>\lambda^{-\frac{1}{2}}\right\}$. Since $f \in W^{1,2}\left(\Omega, \mathcal{A}_{Q}\right)$, for $\lambda \rightarrow 0$, we have that

$$
\operatorname{Dir}(\lambda f, \Omega)=\operatorname{Dir}\left(\lambda f, A_{\lambda}\right)+\lambda^{2} \int_{B_{\lambda}}|D f|^{2}=\operatorname{Dir}\left(\lambda f, A_{\lambda}\right)+o\left(\lambda^{2}\right) .
$$

Using the inequality $\sqrt{1+x^{2}} \geq 1+\frac{x^{2}}{2}-\frac{x^{4}}{4}$ for $|x| \leq 2$, since $\lambda|D f| \leq \sqrt{\lambda}$ in $A_{\lambda}$, for $\lambda \leq 4$ we infer that

$$
\begin{aligned}
\mathbf{M}\left(T_{\lambda f, \Omega}\right) & \geq \sum_{i} \int_{\Omega} \sqrt{1+\lambda^{2}\left|D f_{i}\right|^{2}} \geq Q\left|B_{\lambda}\right|+\int_{A_{\lambda}}\left(1+\frac{\lambda^{2}|D f|^{2}}{2}-C \lambda^{4}|D f|^{4}\right) \\
& \geq Q|\Omega|+\frac{\lambda^{2}}{2} \operatorname{Dir}\left(f, A_{\lambda}\right)-\int_{A_{\lambda}} C \lambda^{3}|D f|^{2} \\
& \stackrel{(2.6)}{=} Q|\Omega|+\frac{\lambda^{2}}{2} \operatorname{Dir}(f, \Omega)+o\left(\lambda^{2}\right) .
\end{aligned}
$$

For what concerns the reversed inequality, we argue as follows. In $A_{\lambda}$, since for every multi index $\alpha$ with $|\alpha| \geq 2$ we have

$$
\lambda^{2|\alpha|}\left|M_{f_{i}}^{\alpha}\right|^{2} \leq C \lambda^{2|\alpha|}\left|D f_{i}\right|^{2|\alpha|} \leq C \lambda^{3}\left|D f_{i}\right|^{2},
$$


we use the inequality $\sqrt{1+x^{2}} \leq 1+\frac{x^{2}}{2}$ and get

$$
\begin{aligned}
\mathbf{M}\left(T_{\lambda f, A_{\lambda}}\right) & \leq \sum_{i} \int_{A_{\lambda}} \sqrt{1+\lambda^{2}\left|D f_{i}\right|^{2}+C \lambda^{3}\left|D f_{i}\right|^{2}} \\
& =Q\left|A_{\lambda}\right|+\frac{\lambda^{2}}{2} \operatorname{Dir}\left(f, A_{\lambda}\right)+o\left(\lambda^{2}\right) .
\end{aligned}
$$

In $B_{\lambda}$, instead, we use the same inequality and the condition $\mathbf{M}\left(T_{f, \Omega}\right)<+\infty$ to infer

$$
\begin{aligned}
\mathbf{M}\left(T_{\lambda f, B_{\lambda}}\right) & \leq \sum_{i} \int_{B_{\lambda}} \sqrt{1+\lambda^{2}\left|D f_{i}\right|^{2}}+\sqrt{\sum_{|\alpha| \geq 2} \lambda^{2|\alpha|} M_{f_{i}}^{\alpha 2}} \\
& \leq Q\left|B_{\lambda}\right|+\frac{\lambda^{2}}{2} \operatorname{Dir}\left(f, B_{\lambda}\right)+\sum_{i} \int_{B_{\lambda}} \lambda^{2} \sqrt{\sum_{|\alpha| \geq 2} M_{f_{i}}^{\alpha 2}} \\
& \stackrel{(2.6)}{\leq} Q\left|B_{\lambda}\right|+o\left(\lambda^{2}\right)+\lambda^{2} \mathbf{M}\left(T_{f, B_{\lambda}}\right)=Q\left|B_{\lambda}\right|+o\left(\lambda^{2}\right) .
\end{aligned}
$$

From (2.7), (2.8) and (2.9), the proof follows.

\section{Complex varieties And Dir-Minimizing FunCtions}

3.1. Complex varieties as minimal currents. In the following we consider irreducible holomorphic varieties $\mathscr{V} \subseteq \mathbb{C}^{\mu+\nu}$ of dimension $\mu$. Following Federer [6], we associate to $\mathscr{V}$ the integer rectifiable current of real dimension $2 \mu$ denoted by $\llbracket \mathscr{V} \rrbracket$ given by the integration over the manifold part of $\mathscr{V}, \mathscr{V}_{\text {reg. }}$. Recall that the singular part $\mathscr{V}_{\text {sing }}=\mathscr{V} \backslash \mathscr{V}_{\text {reg }}$ is a complex variety of dimension at most $(\mu-1)$. A well-known result by Federer asserts that $\llbracket \mathscr{V} \rrbracket$ is a mass-minimizing cycle.

Theorem 3.1. Let $\mathscr{V}$ be an irreducible holomorphic variety. Then, the integer

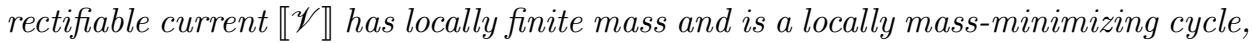
that means $\partial \llbracket \mathscr{V} \rrbracket=0$ and $\mathbf{M}(\llbracket \mathscr{V} \rrbracket) \leq \mathbf{M}(S)$ for every integer current $S$ with $\partial S=0$ and $\operatorname{supp}(S-\llbracket \mathscr{V} \rrbracket)$ compact.

We consider domains $\Omega \subseteq \mathbb{R}^{2 \mu} \simeq \mathbb{C}^{\mu}$ with the usual identification $\left(x_{l}, y_{l}\right) \simeq z_{l}=$ $\left(x_{l}+i y_{l}\right)$ for $l=1, \ldots, \mu$. Moreover, $\mathscr{V} \subseteq \Omega \times \mathbb{R}^{2 \nu} \subseteq \mathbb{R}^{2 \mu+2 \nu} \simeq \mathbb{C}^{\mu+\nu}$ is always supposed to be a $Q$ : 1-cover of $\Omega$ under the orthogonal projection $\pi$ onto $\Omega$, that

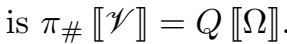

Clearly, under this hypothesis, there exists a $Q$-valued function $f: \Omega \rightarrow \mathcal{A}_{Q}\left(\mathbb{R}^{2 \nu}\right)$ such that $\mathscr{V}=\operatorname{graph}(f)$. From Definition 1.3 , we readily deduce $\Sigma_{f} \subseteq \pi\left(\mathscr{V}_{\text {sing }}\right)$, which in particular implies $\operatorname{dim}_{\mathcal{H}}\left(\Sigma_{f}\right) \leq 2 \mu-2$. Therefore, locally in $\Omega \backslash \Sigma_{f} \times \mathbb{R}^{2 \nu}, \mathscr{V}$ is the superposition of graphs of holomorphic functions, that is, for every $w \in \Omega \backslash \Sigma_{f}$, there exist a radius $r$ and $Q$ holomorphic functions $f_{i}: B_{r}(w) \rightarrow \mathbb{C}^{\nu}$ such that $\left.f\right|_{B_{r}(w)}=\sum_{i} \llbracket f_{i} \rrbracket$. The following are the main properties of $f$.

Proposition 3.2. Let $\mathscr{V} \subseteq \Omega \times \mathbb{R}^{2 \nu}$ be a holomorphic variety as above and $f$ the associated $Q$-valued function. Then, the following hold:

(i) $f \in W^{1,2}\left(\Omega, \mathcal{A}_{Q}\right)$ and, for $\mu=1, M\left(\llbracket \mathscr{V} \rrbracket\llcorner\Omega)=Q+\frac{\operatorname{Dir}(f, \Omega)}{2}\right.$;

(ii) $\llbracket \mathscr{V} \rrbracket\left\llcorner\Omega=T_{f, \Omega}\right.$ and $\partial\left(\llbracket \mathscr{V} \rrbracket\left\llcorner B_{r}(x)\right)=T_{f, \partial B_{r}(x)}\right.$ for every $x$ and a.e. $r>0$ with $B_{r}(x) \subseteq \Omega$. 
Proof. Note that, for every smooth $h: \mathbb{R}^{2} \rightarrow \mathbb{R}^{2 \nu}$ and, as usual, $\bar{h}(w)=(w, h(w))$,

$$
\sqrt{\operatorname{det}\left(D \bar{h} \cdot D \bar{h}^{T}\right)} \leq 1+\frac{|D h|^{2}}{2},
$$

with equality if and only if $h$ is conformal, i.e. $\left|\partial_{x} h\right|=\left|\partial_{y} h\right|$ and $\partial_{x} h \cdot \partial_{y} h=0$. Indeed, (3.1) reads as

$$
\operatorname{det}\left(D \bar{h} \cdot D \bar{h}^{T}\right)=\operatorname{det}\left(\begin{array}{cc}
1+\left|\partial_{x} h\right| & \partial_{x} h \cdot \partial_{y} h \\
\partial_{x} h \cdot \partial_{y} h & 1+\left|\partial_{y} h\right|
\end{array}\right) \leq\left(1+\frac{\left|\partial_{x} h\right|^{2}+\left|\partial_{y} h\right|^{2}}{2}\right)^{2}
$$

which in turn is equivalent to $0 \leq\left(\left|\partial_{x} h\right|^{2}-\left|\partial_{y} h\right|^{2}\right)^{2}+4\left(\partial_{x} h \cdot \partial_{y} h\right)^{2}$.

In the case $\mu=1$, applying (3.1) to the local holomorphic, hence conformal, selection of $f$, from (2.3) we get

$$
M\left(\llbracket \mathscr{V} \rrbracket\left\llcorner\left(\Omega \backslash \Sigma_{f}\right)\right)=Q+\frac{\operatorname{Dir}\left(f, \Omega \backslash \Sigma_{f}\right)}{2} .\right.
$$

In the case $\mu>1$ and $g: \mathbb{R}^{2 \mu} \rightarrow \mathbb{R}^{2 \nu}$ smooth, (3.1) together with Binet-Cauchy's formula (see $[5$, Section 3.2 Theorem 4$]$ ), for every $l=1, \cdots, \mu$, we infer

$$
\begin{aligned}
\operatorname{det}\left(D \bar{g} \cdot D \bar{g}^{T}\right) & =1+|D g|^{2}+\sum_{|\alpha|=|\beta| \geq 2} M_{\alpha \beta}(D g)^{2} \\
& \geq 1+\left|\partial_{x_{l}} g\right|^{2}+\left|\partial_{y_{l}} g\right|^{2}+\sum_{i, j=1}^{2 \nu}\left(\partial_{x_{l}} g^{i} \partial_{y_{l}} g^{j}-\partial_{x_{l}} g^{j} \partial_{y_{l}} g^{i}\right)^{2} \\
& =\operatorname{det}\left(\nabla_{l} \bar{g} \cdot \nabla_{l} \bar{g}^{T}\right),
\end{aligned}
$$

where $M_{\alpha \beta}$ stands for the $\alpha, \beta$ minors of a matrix and $\nabla_{l}$ denotes the derivative with respect to $x_{l}$ and $y_{l}$. Hence, if $f_{i}$ is a local holomorphic, consequently conformal, selection for $f: \Omega \subset \mathbb{R}^{2 \mu} \rightarrow \mathcal{A}_{Q}$, we infer that

$$
\begin{aligned}
\mu Q+\frac{|D f|^{2}}{2} & =\sum_{i=1}^{Q} \sum_{l=1}^{\mu}\left(1+\frac{\left|\nabla_{l} f_{i}\right|^{2}}{2}\right) \stackrel{(3.1)}{=} \sum_{i=1}^{Q} \sum_{l=1}^{\mu} \sqrt{\operatorname{det}\left(\nabla_{l} \bar{f}_{i} \cdot \nabla_{l} \bar{f}_{i}^{T}\right)} \\
& \stackrel{(3.3)}{\leq} \mu \sum_{i=1}^{Q} \sqrt{\operatorname{det}\left(D \bar{f}_{i} \cdot D \bar{f}_{i}^{T}\right)}
\end{aligned}
$$

Integrating, we conclude, for $\mu>1$,

$$
M\left(\llbracket \mathscr{V} \rrbracket\left\llcorner\left(\Omega \backslash \Sigma_{f}\right)\right) \geq Q+\frac{\operatorname{Dir}\left(f, \Omega \backslash \Sigma_{f}\right)}{2 \mu} .\right.
$$

Now since the mass of $\llbracket \mathscr{V} \rrbracket$ is finite, by (3.2) and (3.4) the energy of $f$ is finite in $\Omega \backslash \Sigma_{f}$. Being $\operatorname{dim}_{\mathcal{H}}\left(\Sigma_{f}\right) \leq m-2$, Lemma 1.1 gives $(i)$.

Being $\llbracket \mathscr{V} \rrbracket$ defined by the integration over $\mathscr{V}_{\text {reg }}$ and $\mathcal{H}^{m}\left(\pi\left(\mathscr{V}_{\text {sing }}\right)\right)=0$, it follows straightforwardly that $T_{f, \Omega}$ is well-defined by $(2.1)$ and coincides with $\llbracket \mathscr{V} \rrbracket$. For the same reason, since also $\mathcal{H}^{m-1}\left(\pi\left(\mathscr{V}_{\text {sing }}\right)\right)=0, \partial\left(\llbracket \mathscr{V} \rrbracket\left\llcorner B_{r}(x)\right)=T_{f, \partial B_{r}(x)}\right.$ for every $B_{r}(x) \subseteq \Omega$ such that $\left.f\right|_{\partial B_{r}(x)} \in W^{1,2}$ and $\mathbf{M}\left(\partial\left(\llbracket \mathscr{V} \rrbracket\left\llcorner B_{r}(x)\right)\right)\right.$ is finite, that is for every $x$ and a.e. $r>0$, thus concluding the proof of $(i i)$. 
3.2. Proof of Theorem 0.1. Now we are ready to prove the first main result of the paper. We divide the proof in two parts: in the first one we give an argument for the planar case which is particularly simple and exploit the equality between the area and the energy functionals; in the second part we give a proof valid in every dimension.

3.2.1. Planar case $\mu=1$. In view of Proposition 3.2, we need only to show that $f$ is Dir-minimizing in $B_{1}$. Choose a radius $r \in[1,2]$ such that $\partial B_{r} \cap \Sigma_{f}=\emptyset$ and set $g=\left.f\right|_{\partial B_{r}}$. Note that $g$ is Lipschitz continuous. For every $h \in \operatorname{Lip}\left(B_{r}, \mathcal{A}_{Q}\right)$ with $\left.h\right|_{\partial B_{r}}=g$, from the Taylor expansion of the mass and from (3.1), we infer that

$$
\mathbf{M}\left(T_{h, B_{r}}\right)-Q \leq \frac{\operatorname{Dir}\left(h, B_{r}\right)}{2} .
$$

By Theorem 2.3, $\partial T_{h, B_{r}}=T_{f, \partial B_{r}}=\partial\left(\llbracket \mathscr{V} \rrbracket\left\llcorner B_{r}\right)\right.$. So, using Theorem 3.1 we infer

$$
\operatorname{Dir}\left(f, B_{r}\right) \stackrel{(3.2)}{=} 2\left(\mathbf{M}\left(T_{f, B_{r}}\right)-Q\right) \leq 2\left(\mathbf{M}\left(T_{h, B_{r}}\right)-Q\right) \stackrel{(3.5)}{\leq} \operatorname{Dir}\left(h, B_{r}\right) .
$$

Since the set of Lipschitz functions with trace $g$ is dense in $W_{g}^{1,2}\left(B_{r}, \mathcal{A}_{Q}\right)$ (see $[4$, Section 14]), this implies that $f$ is Dir-minimizing in $B_{r}$ and, a fortiori, in $B_{1}$.

Remark 3.3. The planar result provides examples of Dir-minimizing functions with singular set of dimension $m-2$ for every $m$, thus proving the optimality of the regularity Theorem 1.4. Indeed, if $g: B_{1} \subseteq \mathbb{R}^{2} \rightarrow \mathcal{A}_{Q}$ is Dir-minimizing and $\Sigma_{g} \neq \emptyset$, then $f: B_{1} \times \mathbb{R}^{m-2} \rightarrow \mathcal{A}_{Q}$ with $f\left(x_{1}, x_{2}, \ldots, x_{m}\right)=g\left(x_{1}, x_{2}\right)$ is also Dir-minimizing (see [4, Lemma 3.24]) and $\operatorname{dim}_{\mathcal{H}}\left(\Sigma_{f}\right)=m-2$.

3.2.2. General case $\mu \geq 1$. Here we exploit the expansion of the mass given in Lemma 2.5. The reason why this can be done without the strong approximation theory developed by Almgren in [1] and reproved with different methods in [3] is that, given as above a complex variety which is the graph of a multi-valued function, the rescaled current $L_{\lambda \#} \llbracket \mathscr{V} \rrbracket=T_{\lambda f}$, where $L_{\lambda}: \mathbb{C}^{\mu+\nu} \rightarrow \mathbb{C}^{\mu+\nu}$ is given by $L_{\lambda}(x, y)=(x, \lambda y)$, is also a complex variety (being the $L_{\lambda}$ 's linear complex maps), and, hence, it is also area-minimizing.

The proof is by contradiction. Assume $f$ is not Dir-minimizing in $B_{1}$. Then, there exists $u \in W^{1,2}\left(B_{1}, \mathcal{A}_{Q}\right)$ and $\eta>0$ such that $\operatorname{Dir}\left(u, B_{1}\right) \leq \operatorname{Dir}\left(f, B_{1}\right)-\eta$ and $\left.u\right|_{\partial B_{1}}=\left.f\right|_{\partial B_{1}}$. Set

$$
w= \begin{cases}u & \text { in } B_{1}, \\ f & \text { in } B_{2} \backslash B_{1} .\end{cases}
$$

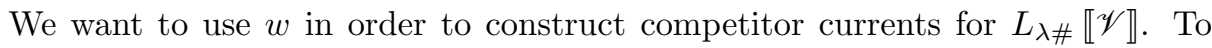
this aim, consider first its Lipschitz approximations $w_{\varepsilon}$, for every $\varepsilon>0$, such that (see [4, Proposition 4.4]):

(a) $\left|E_{\varepsilon}\right|=o\left(\varepsilon^{2}\right)$ as $\varepsilon \rightarrow 0$, where $E_{\varepsilon}=\left\{w_{\varepsilon} \neq w\right\}$;

(b) $\operatorname{Lip}\left(w_{\varepsilon}\right) \leq \varepsilon^{-1}$;

(c) ||$\left|D w_{\varepsilon}\right|-|D w| \|_{L^{2}}=o(1)$ as $\varepsilon \rightarrow 0$.

By Proposition 3.2 and Lemma 2.5, for every open $A$ such that $E_{\varepsilon} \subseteq A$ and $|A| \leq 2\left|E_{\varepsilon}\right|$,

$$
\begin{aligned}
\mathbf{M}\left(L_{\lambda \#}\left(\llbracket \mathscr{V} \rrbracket\left\llcorner\left(E_{\varepsilon} \times \mathbb{R}^{2 \nu}\right)\right)\right)\right. & =\mathbf{M}\left(T_{\lambda f, E_{\varepsilon}}\right) \leq \mathbf{M}\left(T_{\lambda f, A}\right) \\
& \stackrel{(2.5)}{=} Q|A|+\frac{\lambda^{2}}{2} \int_{A}|D f|^{2}+o\left(\lambda^{2}\right)=o\left(\varepsilon^{2}\right)+O\left(\lambda^{2}\right) .
\end{aligned}
$$


Using Fubini and again Proposition 3.2, we can find radii $r_{\lambda, \varepsilon}$ such that

$$
\begin{gathered}
\left|E_{\varepsilon} \cap \partial B_{r_{\lambda, \varepsilon}}\right|=o\left(\varepsilon^{2}\right), \\
\partial\left(L_{\lambda \# \llbracket} \llbracket \mathscr{V} \rrbracket\left\llcorner B_{r}\right)=T_{\lambda f, \partial B_{r}} \quad \text { and } \quad \mathbf{M}\left(T_{\lambda f, E_{\varepsilon} \cap \partial B_{r}}\right)=o\left(\varepsilon^{2}\right)+O\left(\lambda^{2}\right) .\right.
\end{gathered}
$$

Set $S_{\lambda \varepsilon}=T_{\lambda f, \partial B_{r_{\lambda, \varepsilon}}}-T_{\lambda w_{\varepsilon}, \partial B_{r_{\lambda, \varepsilon}}}$. Note that, by Theorem 2.3, being $w_{\varepsilon}$ Lipschitz,

$$
\partial S_{\lambda \varepsilon}=\partial T_{\lambda f, \partial B_{r_{\lambda, \varepsilon}}}-\partial T_{\lambda w_{\varepsilon}, \partial B_{r_{\lambda, \varepsilon}}} \stackrel{(3.7)}{=} \partial \partial\left(L_{\lambda \#} \llbracket \mathscr{V} \rrbracket\left\llcorner B_{r}\right)=0 .\right.
$$

Moreover, since $\operatorname{Lip}\left(\lambda w_{\varepsilon}\right) \leq \lambda \varepsilon^{-1}$ and $T_{\lambda f, \partial B_{r_{\lambda, \varepsilon}} \backslash E_{\varepsilon}}=T_{\lambda w_{\varepsilon}, \partial B_{r_{\lambda, \varepsilon}} \backslash E_{\varepsilon}}$, the mass of $S_{\lambda \varepsilon}$ can be estimated in the following way:

$$
\begin{aligned}
\mathbf{M}\left(S_{\lambda \varepsilon}\right) & =\mathbf{M}\left(T_{\lambda f, E_{\varepsilon} \cap \partial B_{r_{\lambda, \varepsilon}}}\right)+\mathbf{M}\left(T_{\lambda w_{\varepsilon}, E_{\varepsilon} \cap \partial B_{r_{\lambda, \varepsilon}}}\right) \\
& \stackrel{(3.7)}{\leq} o\left(\varepsilon^{2}\right)+O\left(\lambda^{2}\right)+C \frac{\lambda\left|E_{\varepsilon}\right|}{\varepsilon} \stackrel{(3.6)}{\leq} o\left(\varepsilon^{2}\right)+O\left(\lambda^{2}\right)+o(\lambda \varepsilon) .
\end{aligned}
$$

For $\varepsilon=\lambda, \mathbf{M}\left(S_{\lambda \lambda}\right)=O\left(\lambda^{2}\right)$ and, by the isoperimetric inequality [8, Theorem 30.1], there exists an integer current $R_{\lambda}$ such that

$$
\partial R_{\lambda}=S_{\lambda \lambda} \quad \text { and } \quad \mathbf{M}\left(R_{\lambda}\right) \leq \mathbf{M}\left(S_{\lambda \lambda}\right)^{\frac{m}{m-1}}=o\left(\lambda^{2}\right) .
$$

The current $T_{\lambda}=T_{\lambda w_{\lambda}, B_{r_{\lambda}}}+R_{\lambda}$ contradicts now the minimality of the complex current $L_{\lambda \#}\left(\llbracket \mathscr{V} \rrbracket\left\llcorner B_{r_{\lambda}}\right)\right.$. Indeed, it is easy to verify that $\partial T_{\lambda}=\partial\left(L_{\lambda \#} \llbracket \mathscr{V} \rrbracket\left\llcorner B_{r_{\lambda}}\right)\right.$ and, for small $\lambda$,

$$
\begin{aligned}
& \mathbf{M}\left(T_{\lambda}\right)-\mathbf{M}\left(L_{\lambda \#} \llbracket \mathscr{V} \rrbracket\left\llcorner\left(B_{r_{\lambda}} \times \mathbb{R}^{2 \nu}\right)\right)=Q\left|B_{r_{\lambda}}\right|+\frac{\lambda^{2}}{2} \operatorname{Dir}\left(w_{\lambda}, B_{r_{\lambda}}\right)+\right. \\
& -Q\left|B_{r_{\lambda}}\right|-\frac{\lambda^{2}}{2} \operatorname{Dir}\left(f, B_{r_{\lambda}}\right)+o\left(\lambda^{2}\right) \\
& \leq-\frac{\lambda^{2} \eta}{4}+o\left(\lambda^{2}\right)<0 \text {. }
\end{aligned}
$$

\section{Higher integrability of the Gradients of Dir-minimizing functions}

In this section we prove Theorem 0.2. As above, for the planar case we give a simple proof which in addition provides the optimal integrability exponent. This proof relies on the following proposition, because by Theorem 1.4 the singular points are isolated in dimension two.

Proposition 4.1. Let $u \in W^{1,2}\left(B_{2}, \mathcal{A}_{Q}\right)$ be Dir-minimizing and assume that $\Sigma_{u}=$ $\{0\}$. Then, $|D u| \in L^{p}\left(B_{1}\right)$ for every $p<\frac{2 Q}{Q-1}$.

Proof. Let $x \in B_{1} \backslash\{0\}$ and set $r=|x|$. Then, by $\Sigma_{u}=\{0\}$, in $B_{r}(x)$ there exists an analytic selection of $u,\left.u\right|_{B_{r}(x)}=\sum_{i} \llbracket u_{i} \rrbracket$, where $u_{i}: B_{r}(x) \rightarrow \mathbb{R}^{n}$ are harmonic functions. Using the mean value inequality for $D u_{i}$, one infers that

$$
\left|D u_{i}(x)\right| \leq f_{B_{r}(x)}\left|D u_{i}\right| \leq \frac{1}{\sqrt{\pi} r}\left(\int_{B_{r}(x)}\left|D u_{i}\right|^{2}\right)^{\frac{1}{2}},
$$

from which

$$
|D u|(x)^{2}=\sum_{i}\left|D u_{i}(x)\right|^{2} \leq \frac{1}{\pi r^{2}} \sum_{i} \int_{B_{r}(x)}\left|D u_{i}^{2}\right|=\frac{\operatorname{Dir}\left(u, B_{r}(x)\right)}{\pi r^{2}} .
$$


Using the decay estimate (1.3) with $\rho=1$ together with (4.1), we deduce that

$$
|D u|(x) \leq \frac{\operatorname{Dir}\left(u, B_{2}\right)}{\sqrt{\pi} r^{1-\frac{1}{Q}}},
$$

which in turn implies the conclusion,

$$
\int_{B_{1}}|D u|^{p} \leq C \int_{B_{1}} \frac{1}{|x|^{p-\frac{p}{Q}}}<+\infty, \quad \forall p<\frac{2 Q}{Q-1} .
$$

Remark 4.2. The bound $\frac{2 Q}{Q-1}$ for the integrability exponent is optimal. Consider, indeed, the complex variety $\mathscr{V}_{Q}=\left\{(z, w): w^{Q}=z\right\} \subseteq \mathbb{C}^{2}$. By Theorem 0.1, the $Q$-valued function $u(z)=\sum_{w^{Q}=z} \llbracket w \rrbracket$ is Dir-minimizing in $B_{2}$. Moreover, $|D u|(z)=Q|z|^{\frac{1}{Q}-1}$. Hence, $|D u| \in L^{p}$ for every $p<\frac{2 Q}{Q-1}$ and $|D u| \notin L^{\frac{2 Q}{Q-1}}$.

Now we pass to the proof of Theorem 0.2 for $m \geq 3$. The first step is a Caccioppoli's inequality for Dir-minimizing functions. For $P \in \mathbb{R}^{n}$, we denote by $\tau_{P}$ the following map: $\tau_{P}: \mathcal{A}_{Q}\left(\mathbb{R}^{n}\right) \rightarrow \mathcal{A}_{Q}\left(\mathbb{R}^{n}\right)$,

$$
\tau_{P}(T):=\sum_{i} \llbracket T_{i}-P \rrbracket, \quad \text { for every } \quad T=\sum_{i} \llbracket T_{i} \rrbracket .
$$

Lemma 4.3 (Caccioppoli's inequality). Let $u \in W^{1,2}\left(\Omega, \mathcal{A}_{Q}\right)$ be Dir-minimizing. Then, for every $P \in \mathbb{R}^{n}$ and every $\eta \in C_{c}^{\infty}(\Omega)$,

$$
\int_{\Omega}|D u|^{2} \eta^{2} \leq \int_{\Omega}\left|\tau_{P} u\right|^{2}|D \eta|^{2}
$$

In particular, in the case $\Omega=B_{2 r}$,

$$
\int_{B_{\frac{3 r}{2}}}|D u|^{2} \leq \frac{4}{r^{2}} \int_{B_{2 r}}\left|\tau_{P} u\right|^{2} .
$$

Proof. Recall the outer variation [4, Proposition 3.1] for Dir-minimizing functions, $0=\int \sum_{i}\left\langle D f_{i}(x): D_{x} \psi\left(x, f_{i}(x)\right)\right\rangle d x+\int \sum_{i}\left\langle D f_{i}(x): D_{y} \psi\left(x, f_{i}(x)\right) \cdot D f_{i}(x)\right\rangle d x$, and apply it to $\psi(x, y)=\eta(x)^{2}(y-P)$, where $P$ and $\eta$ are as in the statement. Since $D_{x} \psi(x, y)=2 \eta(x) D \eta(x) \otimes(y-P)$ and $D_{y} \psi(x, y)=\eta(x)^{2} \operatorname{Id}_{n}$, this leads to

$$
0=\int_{\Omega} \sum_{i}\left\langle D u_{i}(x): 2 \eta D \eta \otimes\left(u_{i}-P\right)\right\rangle+\int_{\Omega} \sum_{i}\left\langle D u_{i}(x): \eta^{2} D u_{i}(x)\right\rangle .
$$

Applying Hölder's inequality in (4.4), we conclude (4.2):

$$
\begin{aligned}
\int_{\Omega} \eta^{2}|D u|^{2} & =-\sum_{i} \int_{\Omega}\left\langle D u_{i} \cdot\left(u_{i}-P\right), \eta D \eta\right\rangle \leq \int_{\Omega} \sum_{i}\left|D u_{i}\right|\left|u_{i}-P\right||\eta||D \eta| \\
& \leq \int_{\Omega}\left(\sum_{i}\left|D u_{i}\right|^{2}|\eta|^{2}\right)^{\frac{1}{2}}\left(\sum_{i}\left|u_{i}-P\right||D \eta|^{2}\right)^{\frac{1}{2}} \\
& \leq\left(\int_{\Omega} \eta^{2}|D u|^{2}\right)^{\frac{1}{2}}\left(\int_{\Omega}\left|\tau_{P}(u)\right|^{2}|D \eta|^{2}\right)^{\frac{1}{2}} .
\end{aligned}
$$

The last conclusion of the lemma follows from (4.2) choosing $\eta \equiv 1$ in $B_{3 r / 2}$ and $|D \eta| \leq \frac{2}{r}$. 
The following reverse Hölder inequality is the basic estimate for the higher integrability.

Proposition 4.4. Let $\frac{2 m}{m+2}<s<2$. Then, there exists $C>0$ such that, for every $u: \Omega \rightarrow \mathcal{A}_{Q}$ Dir-minimizing, $x \in \Omega$ and $r<\min \{1, \operatorname{dist}(x, \partial \Omega) / 2\}$,

$$
\left(f_{B_{r}(x)}|D u|^{2}\right)^{\frac{1}{2}} \leq C\left(f_{B_{2 r}(x)}|D u|^{s}\right)^{\frac{1}{s}} .
$$

Proof. The proof is divided into two steps.

Step 1: we assume that $u$ has average $0, \boldsymbol{\eta} \circ u=\frac{\sum_{i} u_{i}}{Q}=0$.

The proof is by induction on the number of values $Q$. The basic step $Q=1$ is clear: indeed, in this case $\boldsymbol{\eta} \circ u=u=0$. Now, we assume that (4.5) holds for every $Q^{\prime}<Q$ and, by contradiction, it does not hold for $Q$.

Then, up to translations and dilations of the domain, there exists a sequence $\left(u_{l}\right)_{l} \subset W^{1,2}\left(B_{4}, \mathcal{A}_{Q}\right)$ of Dir-minimizing functions such that $\boldsymbol{\eta} \circ u_{l}=0$ and

$$
\left(f_{B_{4}}\left|D u_{l}\right|^{s}\right)^{\frac{1}{s}}<\frac{\left(f_{B_{2}}\left|D u_{l}\right|^{2}\right)^{\frac{1}{2}}}{l} .
$$

Moreover, without loss of generality, we may also assume that $\int_{B_{4}}\left|u_{l}\right|^{2}=1$. Using Caccioppoli's inequality (4.3), we have that $\operatorname{Dir}\left(u_{l}, B_{3}\right) \leq 4$, which in turn, by (4.6), implies

$$
\left\|\mathcal{G}\left(u_{l}, Q \llbracket 0 \rrbracket\right)\right\|_{W^{1, s}\left(B_{4}\right)} \leq C<+\infty .
$$

Since $s^{*}>2$, we can apply the compact Sobolev embedding (see [4, Proposition 2.11]) to deduce that there exists a subsequence (not relabeled) $u_{l}$ converging to some $u$ in $L^{2}\left(B_{4}\right)$. From (4.6) and Lemma 1.2, we deduce that

$$
\int_{B_{4}}|u|^{2}=1 \quad \text { and } \quad \int_{B_{4}}|D u|^{s}=0
$$

which implies that $u$ is constant, $u \equiv T \in \mathcal{A}_{Q}$. Since by Theorem 1.4 the $u_{l}$ 's are equi-bounded and equi-Hölder in $B_{2}$, always up to a subsequence (again not relabeled), the $u_{l}^{\prime} s$ converge uniformly to $T$ in $B_{2}$. This implies, in particular, that

$$
\boldsymbol{\eta} \circ T=\lim _{l \rightarrow+\infty} \boldsymbol{\eta} \circ u_{l}=0 .
$$

From (4.7) and (4.8), one infers that $T$ is not a point of multiplicity $Q$. Therefore, since $u_{l} \rightarrow T$ uniformly in $B_{2}$, for $l$ large enough the $u_{n}$ 's must split in the sum of two Dir-minimizing functions $u_{l}=\llbracket v_{l} \rrbracket+\llbracket w_{l} \rrbracket$, where the $v_{l}$ 's are $Q_{1}$-valued functions and the $w_{l}$ 's are $Q_{2}$-valued, with $Q_{1}, Q_{2}$ positive and $Q_{1}+Q_{2}=Q$. Applying now the inductive hypothesis to $v_{l}$ and $w_{l}$ we contradict (4.6) for $l$ large 
enough,

$$
\begin{aligned}
\left(f_{B_{1}(x)}\left|D u_{l}\right|^{2}\right)^{\frac{1}{2}} & \leq\left(f_{B_{1}(x)}\left|D v_{l}\right|^{2}\right)^{\frac{1}{2}}+\left(f_{B_{1}(x)}\left|D w_{l}\right|^{2}\right)^{\frac{1}{2}} \\
& \leq C\left(f_{B_{2}(x)}\left|D v_{l}\right|^{s}\right)^{\frac{1}{s}}+C\left(f_{B_{2}(x)}\left|D w_{l}\right|^{s}\right)^{\frac{1}{s}} \\
& \leq 2 C\left(f_{B_{2}(x)}\left|D u_{l}\right|^{s}\right)^{\frac{1}{s}} .
\end{aligned}
$$

Step 2: generic Dir-minimizing function $u$.

Let $u$ be Dir-minimizing and $\varphi=\boldsymbol{\eta} \circ u$ : then, by [4, Lemma 3.23], $\varphi: \Omega \rightarrow \mathbb{R}^{n}$ is harmonic and $D \varphi=\sum_{i} D u_{i}$, from which

$$
|D \varphi|^{2} \leq Q \sum_{i}\left|D u_{i}\right|^{2}=Q|D u|^{2} .
$$

Moreover, again by [4, Lemma 3.23], the $Q$-valued function $v=\sum_{i} \llbracket u_{i}-\varphi \rrbracket$ is Dir-minimizing as well. Note that

$$
|D u|^{2} \leq 2|D v|^{2}+2 Q|D \varphi|^{2} \quad \text { and } \quad|D v|^{2} \leq 2|D u|^{2}+2 Q|D \varphi|^{2} .
$$

Using the inequality $\sqrt{\sum_{j} a_{j}} \leq \sum_{j} \sqrt{a_{j}}$ for positive $a_{j}$, we deduce

$$
\begin{aligned}
\left(f_{B_{r}(x)}|D u|^{2}\right)^{\frac{1}{2}} & \leq\left(f_{B_{r}(x)} 2|D v|^{2}+2 Q|D \varphi|^{2}\right)^{\frac{1}{2}} \\
& \leq 2\left(f_{B_{r}(x)}|D v|^{2}\right)^{\frac{1}{2}}+2 Q\left(f_{B_{r}(x)}|D \varphi|^{2}\right)^{\frac{1}{2}} .
\end{aligned}
$$

For the first term in the right hand side of (4.11), we use Step 1, since $\boldsymbol{\eta} \circ v=0$, to get

$$
\begin{aligned}
\left(f_{B_{r}(x)}|D v|^{2}\right)^{\frac{1}{2}} & \leq C\left(f_{B_{2 r}(x)}|D v|^{s}\right)^{\frac{1}{s}} \stackrel{(4.10)}{\leq} C\left(f_{B_{2 r}(x)}\left(2|D u|^{2}+2 Q|D \varphi|^{2}\right)^{\frac{s}{2}}\right)^{\frac{1}{s}} \\
& \leq C\left(f_{B_{2 r}(x)} 2|D u|^{s}+2 Q|D \varphi|^{s}\right)^{\frac{1}{s}} \stackrel{(4.9)}{\leq} C\left(f_{B_{2 r}(x)}|D u|^{s}\right)^{\frac{1}{s}}
\end{aligned}
$$

For the remaining term in (4.11), we use the standard estimate for harmonic functions,

$$
|D \varphi(x)| \leq \frac{C}{r^{n}}\|D \varphi\|_{L^{1}\left(B_{2 r}\right)} \quad \forall x \in B_{r},
$$


and infer

$$
\begin{aligned}
\left(f_{B_{r}(x)}|D \varphi|^{2}\right)^{\frac{1}{2}} & \stackrel{(4.13)}{\leq} \frac{C}{r^{n}}\|D \varphi\|_{L^{1}\left(B_{2 r}\right)} \leq \frac{C}{r^{n}}\left(\int_{B_{2 r}(x)}|D \varphi|^{s}\right)^{\frac{1}{s}} r^{n\left(1-\frac{1}{s}\right)} \\
& \leq C\left(f_{B_{2 r}(x)}|D \varphi|^{s}\right)^{\frac{1}{s}} \stackrel{(4.9)}{\leq} C\left(f_{B_{2 r}(x)}|D u|^{s}\right)^{\frac{1}{s}}
\end{aligned}
$$

Clearly, (4.11), (4.12) and (4.14) finish the proof.

The proof of Theorem 0.2 is now an easy consequence of the following reversed Hölder inequality with increasing supports proved by Giaquinta and Modica in [7, Proposition 5.1].

Theorem 4.5 (Reversed Hölder inequality). Let $\Omega \subseteq \mathbb{R}^{m}$ be open and $g \in L_{\text {loc }}^{q}(\Omega)$, with $q>1$ and $g \geq 0$. Assume that there exist positive constants $b$ and $R$ such that

$$
\left(f_{B_{r}(x)} g^{q}\right)^{\frac{1}{q}} \leq b f_{B_{2 r}(x)} g, \quad \forall x \in \Omega, \forall r<\min \{R, \operatorname{dist}(x, \partial \Omega) / 2\} .
$$

Then, there exist $p=p(q, b)>q$ and $c=c(m, q, b)$ such that $g \in L_{l o c}^{p}(\Omega)$ and

$$
\left(f_{B_{r}(x)} g^{p}\right)^{\frac{1}{p}} \leq c\left(f_{B_{2 r}(x)} g^{q}\right)^{\frac{1}{q}}, \quad \forall x \in \Omega, \forall r<\min \{R, \operatorname{dist}(x, \partial \Omega) / 2\} .
$$

Proof of Theorem 0.2. Consider the function $g=|D u|^{s}$, where $s<2$ is the exponent in Proposition 4.4. Estimate (4.5) implies that hypothesis (4.15) of Theorem 4.5 is satisfied with $q=\frac{2}{s}>1$. Hence, there exists an exponent $p^{\prime}>q$, such that $g$ belongs to $L_{l o c}^{p^{\prime}}(\Omega)$, i.e. $|D u| \in L_{l o c}^{p}(\Omega)$ for $p=p^{\prime} \cdot s>2$.

\section{REFERENCES}

[1] F. J. Almgren, Jr. Almgren's big regularity paper, volume 1 of World Scientific Monograph Series in Mathematics. World Scientific Publishing Co. Inc., River Edge, NJ, 2000. Q-valued functions minimizing Dirichlet's integral and the regularity of area-minimizing rectifiable currents up to codimension 2, With a preface by Jean E. Taylor and Vladimir Scheffer.

[2] C. De Lellis, M. Focardi, and E. N. Spadaro. Quasi-convexity and semicontinuity of $Q-$ integrands. Preprint, 2009.

[3] C. De Lellis and E. N. Spadaro. Higher integrability and approximation of minimal currents. 2009.

[4] C. De Lellis and E. N. Spadaro. Q-valued functions revisited. Accepted for Memoirs AMS, 2009.

[5] L. C. Evans and R. F. Gariepy. Measure theory and fine properties of functions. Studies in Advanced Mathematics. CRC Press, Boca Raton, FL, 1992.

[6] H. Federer. Some theorems on integral currents. Trans. Amer. Math. Soc., 117:43-67, 1965.

[7] M. Giaquinta and G. Modica. Regularity results for some classes of higher order nonlinear elliptic systems. J. Reine Angew. Math., 311/312:145-169, 1979.

[8] L. Simon. Lectures on geometric measure theory, volume 3 of Proceedings of the Centre for Mathematical Analysis, Australian National University. Australian National University Centre for Mathematical Analysis, Canberra, 1983.

UNIVERSITÄT ZÜRICH

E-mail address: emanuele.spadaro@math.uzh.ch 